\title{
Paediatric empyema: are ultrasound characteristics at the time of intervention predictive of treatment failure?
}

\author{
Stuart Haggie $^{1}$, Hiran Selvadurai ${ }^{2}$, Hasantha Gunasekera ${ }^{1}$, Dominic Fitzgerald ${ }^{3}$, David \\ Lord $^{1}$, and Murthy Chennapragada ${ }^{1}$ \\ ${ }^{1}$ Children's Hospital at Westmead \\ ${ }^{2}$ The Childrenś Hospital at Westmead \\ ${ }^{3}$ The Children's Hospital at Westmead
}

October 28, 2021

\begin{abstract}
Background: Parapneumonic effusions and empyema are the most frequent complication of paediatric pneumonia. Treatment options include chest drain and fibrinolytics (CDF) or thoracoscopic surgery. CDF is considered less invasive, more cost effective though with higher rates of reintervention. Pleural fluid characteristics on ultrasound may identify cases at increased risk of treatment failure with primary CDF. Methods: A retrospective cohort 2011-2018 of complicated pneumonia managed with primary CDF. Cases were reviewed using ultrasound criteria to describe pleural fluid. We compared ultrasound findings and treatment failure. Results: We report 137 cases with a median age 3.8 years and $43 \%$ female. Treatment failure occurred for $32 / 137(23 \%)$ cases. The interobserver reliability was substantial for the number of septations (Kappa $0.72,95 \%$ CI 0.6 to 0.8 ), moderate for the size of the largest locule (Kappa 0.55, 95\% CI 0.4 to 0.7 ) and fair for the level of echogenicity (Kappa $0.22,95 \%$ CI 0.1 to 0.3 ), pleural thickening (Kappa 0.28, 95\% CI 0.2 to 0.4), maximum effusion depth (Kappa 0.37, 95\%CI 0.2 to 0.5 ) and radiologist's risk for reintervention (Kappa 0.32, 95\% CI 0.2 to 0.5 ). Hyperechoic pleural fluid was associated with treatment failure, with cases nearly five times more likely than anechoic fluid to have a reintervention (OR 4.9 95\%CI 1.7 to $14.2, \mathrm{p}=0.04)$. Treatment failure was not associated with other variables. Conclusion: We did not find an association between ultrasound characteristics and treatment failure for complicated pneumonia managed with primary CDF. Inter-observer agreement of ultrasound findings was difficult despite more objective criteria.
\end{abstract}

Paediatric empyema: are ultrasound characteristics at the time of intervention predictive of treatment failure?

Stuart Haggie ${ }^{1,2}$ MBBS, FRACP, Hiran Selvadurai ${ }^{1,2}$ MBBS, FRACP, PhD, Hasantha Gunasekera ${ }^{2}$ MBBS, DCH, MIPH (Hons), FRACP, PhD, Dominic A. Fitzgerald ${ }^{1,2}$ MBBS, FRACP, PhD, David Lord MBBS, FRANZCR $^{2,3}$, S.Murthy Chennapragada MBBS, FRANZCR ${ }^{2,3}$

${ }^{1}$ Department of Respiratory Medicine, The Children's Hospital at Westmead, Sydney, NSW, Australia, 2145

${ }^{2}$ Children's Hospital Westmead Clinical School, Faculty of Medicine and Health, The University of Sydney, NSW, Australia, 2145

${ }^{3}$ Paediatric Interventional Radiology, Division of Medical Imaging, The Children's Hospital at Westmead, Sydney, NSW, Australia, 2145

Financial Disclosure : The authors have no financial relationships relevant to this article to disclose.

Keywords : Imaging, Infections: Pneumonia 
Corresponding author : Dr. Stuart Haggie, Department of Respiratory Medicine, The Children's Hospital Westmead, Sydney, NSW, Australia, 2145. +6129845 0000. stuart.haggie@health.nsw.gov.au

Short Title: Ultrasound and empyema treatment failure

\section{Abstract:}

Background: Parapneumonic effusions and empyema are the most frequent complication of paediatric pneumonia. Treatment options include chest drain and fibrinolytics (CDF) or thoracoscopic surgery. CDF is considered less invasive, more cost effective though with higher rates of reintervention. Pleural fluid characteristics on ultrasound may identify cases at increased risk of treatment failure with primary CDF.

Methods: A retrospective cohort 2011-2018 of complicated pneumonia managed with primary CDF. Cases were reviewed using ultrasound criteria to describe pleural fluid. We compared ultrasound findings and treatment failure.

Results: We report 137 cases with a median age 3.8 years and $43 \%$ female. Treatment failure occurred for $32 / 137(23 \%)$ cases. The interobserver reliability was substantial for the number of septations (Kappa 0.72, 95\% CI 0.6 to 0.8), moderate for the size of the largest locule (Kappa 0.55, 95\% CI 0.4 to 0.7) and fair for the level of echogenicity (Kappa 0.22, 95\%CI 0.1 to 0.3), pleural thickening (Kappa 0.28, 95\% CI 0.2 to 0.4), maximum effusion depth (Kappa 0.37, 95\% CI 0.2 to 0.5) and radiologist's risk for reintervention (Kappa $0.32,95 \%$ CI 0.2 to 0.5 ). Hyperechoic pleural fluid was associated with treatment failure, with cases nearly five times more likely than anechoic fluid to have a reintervention (OR $4.995 \%$ CI 1.7 to 14.2, $\mathrm{p}=0.04$ ). Treatment failure was not associated with other variables.

Conclusion: We did not find an association between ultrasound characteristics and treatment failure for complicated pneumonia managed with primary CDF. Inter-observer agreement of ultrasound findings was difficult despite more objective criteria.

\section{Background :}

Parapneumonic effusion and empyema thoracis are the most common complications of paediatric community acquired pneumonia. ${ }^{1}$ Distinguishing parapneumonic effusions from a empyema is difficult and thoracocentesis is not recommended in pleural effusions associated with infection. ${ }^{4}$ Without pleural fluid microscopy, biochemistry and culture results, the clinician's ability to precisely discern a parapneumonic effusion from empyema is limited. An invasive drainage procedure is considered for patients with respiratory compromise, a continued fever or an enlarging pleural collection despite optimised medical management (supplemental oxygen and intravenous antibiotics) ${ }^{4}$ reported rates of intervention range from $26-78 \%{ }^{2,3}$

Primary invasive treatment options include chest drainage and fibrinolytic therapy (CDF) or video assisted thoracoscopic surgery (VATS). Since 2006 four randomised controlled trials comparing primary CDF and VATS for the management of paediatric empyema have reported no difference in clinical outcomes (length of stay and procedural reintervention rate) ${ }^{5-8}$ However, a recent meta-analysis including these trials as well as several large retrospective cohorts, has reported a $15 \%$ higher reintervention rate in cases treated with primary CDF vs. VATS (23.9\% vs $8.7 \%) .{ }^{9} \mathrm{CDF}$ is preferred where possible as it is less invasive and more cost effective than VATS. ${ }^{10}$ Over the last 10 years, a trend toward increasing CDF and decreasing VATS as the primary intervention for paediatric empyema has been reported. ${ }^{11}$ Further research is needed to better select cases at high risk of reintervention following CDF, ultrasound assessment of pleural fluid may support clinicians in deciding between these interventions.

Ultrasound is a core imaging modality in the management of complicated pneumonia, it is fast, non-ionizing, portable and inexpensive tool able to confirm the diagnosis of a pleural effusion and guide pleural catheter insertion to the most favourable drainage site. ${ }^{4,12,13}$ A 2009 review of paediatric pleural effusion imaging concluded that ultrasound cannot accurately predict the Light's stage (Exudative, Fibrinopurulent or Organised) of a pleural collection. However paediatric data is lacking and the authors concluded ultrasound 
was useful in assessing disease severity. ${ }^{14}$ Prospective clinical trials comparing primary CDF and VATS have included sonographic criteria of pleural fluid to recruit cases despite this evidence gap. ${ }^{5,6,8}$

A prospective randomised trial comparing VATS and CDF for the management of children with empyema reported no association between ultrasound characteristics (fluid echogenicity or the presence of septations) and duration of hospitalisation. ${ }^{15}$ There is currently insufficient data to determine whether ultrasound characteristics can predict treatment failure in primary CDF for paediatric complicated pneumonia.

\section{Aim :}

We postulated treatment failure would be more likely in cases with ultrasound findings consistent with highly organised pleural effusions, managed with primary CDF. We sought to compare treatment failure outcomes with a detailed ultrasound criteria for pleural fluid.

\section{Methods :}

We report a retrospective cohort of paediatric empyema cases, identified by ICD-10 diagnostic and procedural codes, admitted to the largest tertiary paediatric children's hospital in Sydney, Australia from 2011-2018. Each record was assessed for suitability by SH, records were only included with a clinical diagnosis of empyema, referred for primary CDF with no prior drainage procedure and with available ultrasound images. During the study cases routinely received 48 hours intravenous treatment with broad spectrum antibiotics prior to an invasive drainage procedure, unless a more urgent intervention for respiratory compromise was required.

All ultrasounds were performed at our centre, and the last chest ultrasound prior to intervention was reviewed for each case. Ultrasounds were described according to a prior agreed criteria, independently by two senior consultant paediatric interventional radiologists blinded to the subsequent clinical outcome. Ultrasound images were obtained using a combination of sector probes of frequency ranging between $2-9 \mathrm{MHz}$ and linear probes of frequency ranging between 4 to $15 \mathrm{MHz}$. These images were stored on the PACS (Siemens, Erlangen, Germany). The stored images were reviewed and graded according to predefined novel criteria. The criteria was modified from a previously described (largely qualitative) pleural fluid assessment ${ }^{14}$ by expert consensus from the radiologists participating in this study, to include more objective parameters. We described the pleural fluid characteristics of: echogenicity, the number of septations (defined as an echogenic strand like appearance arising from a pleural surface), the presence of loculations (defined as a pocket of fluid bordered by septations), the visceral pleural thickness and the maximal interpleural depth of fluid. Each radiologist also made a subjective assessment of whether they considered the case a high risk for reintervention. The ultrasound criteria are presented in table 1 and demonstrative images in figure 1.

We compared interobserver agreement between radiologists using Cohen's kappa coefficient for dichotomous variables and a weighted kappa statistic for ordinal variables. This approach was used to report disagreement between two raters on an ordinal scale, as disagreement between extreme categories was considered more important than disagreement on neighbouring categories. We used McHugh's descriptions of kappa interobserver reliability. ${ }^{16} \mathrm{~A}$ p value of $<0.05$ was considered statistically significant. We pooled the radiologists' observations for each ultrasound characteristic and compared these to the clinical outcome of reintervention. We used the paired t-test for continuous variables and $?^{2}$ test for categorical variables. Treatment failure was defined as any repeat invasive procedure following primary CDF, which included VATS, thoracoscopy or repeat chest drainage. All analyses were performed in SAS version 9.4 (SAS Institute; Cary, North Carolina, USA).

\section{Results :}

152 cases were identified from ICD-10 codes, 15 cases were excluded from the analysis with $(2 / 15)$ requiring parenchymal drainage for necrotising pneumonia (without an effusion) and (13/15) due to absent or inadequate ultrasound images to review. There were 137 cases in the final analysis. The median age was 3.8 years (IQR $2.0-5.9 y$ ) and $43 \%$ were female. The mean duration of fever at the time of CDF insertion was 7.3 days (range 1-16 days). The baseline characteristics of cases managed with primary CDF alone and those 
with reintervention are presented in table 2. There were no differences between the cohorts. Treatment failure occurred for 32/137 (23\%) cases. The indications for a repeat intervention were: continued fever with evidence of a persisting collection on ultrasound (15/32), nil drain output with a persisting collection on ultrasound (8/32), a post-procedural air leak with respiratory compromise (6/32), dislodged drain $(2 / 32)$ and focal drainage of a parenchymal collection (1/32).

The ultrasound characteristics and kappa score describing interobserver reliability are presented in Table 3. The interobserver reliability was substantial for the number of septations (Kappa 0.72, 95\% CI 0.6 to 0.8 ), moderate for the size of the largest locule (Kappa 0.55, 95\% CI 0.4 to 0.7 ) and fair for the level of echogenicity (Kappa 0.22, 95\% CI 0.1 to 0.3), pleural thickening (Kappa 0.28, 95\% CI 0.2 to 0.4), maximum effusion depth (Kappa 0.37, 95\%CI 0.2 to 0.5 ) and radiologist's risk for reintervention (Kappa 0.32, 95\% CI 0.2 to 0.5$)$.

Each radiologist's observations were compared to the clinical outcome of a repeat intervention. Hyperechoic pleural fluid was associated with a repeat intervention, with cases nearly five times more likely than anechoic fluid to have a reintervention (OR $4.995 \%$ CI 1.7 to $14.2, \mathrm{p}=0.036$ ). There was no association between a repeat intervention and the number of septations, the size of the largest locule, a pleural rind, the maximal depth of effusion, nor if either interventional radiologist considered the case overall 'high-risk' for a repeat procedure (all $\mathrm{p}>0.05)$.

\section{Discussion :}

We report one of the largest cohorts of paediatric empyema cases managed with primary CDF and, we believe, the first to compare ultrasound findings with treatment failure. We report a clinically important association with reintervention being nearly 5 times more likely to be associated with hyperechoic than anechoic pleural fluid. This finding may influence primary treatment decisions with VATS preferred to CDF in higher risk cases, however, we found only a fair level of agreement between two experienced paediatric radiologists on this variable.

Our findings do not suggest more organised pleural effusions to be at higher risk of treatment failure with primary CDF treatment. The sonographic features of multiple small locules or septations, and the presence of a pleural rind were not associated with treatment failure. Neither were cases considered 'high-risk' by either interventional radiologist associated with a repeat intervention. These results were not what we expected, although consistent with (ref 15) which showed a similar lack of association between septations and length of stay. There is little consensus in other research of whether sonographic features at intervention can predict treatment failure.

A retrospective cohort of chest drainage (without fibrinolytic treatment) in adults of complicated parapneumonic effusion or empyema identified loculations and larger pleural effusions on ultrasound, to be associated with treatment failure. ${ }^{17}$ A prospective paediatric cohort by Kalfa et al. compared ultrasound characteristics and clinical outcomes after primary thoracoscopy, and reported no association with: pleural fluid echogenicity, loculations or larger effusions and adverse clinical outcomes (longer hospitalisation, surgical difficulty, post-operative complications). However, this study compared an alternate primary treatment with a relatively low reintervention rate $(6 \%)$ and did not define the ultrasound criteria. The main finding from this cohort was the association of a delay to procedure of $>4$ days with adverse clinical outcomes. ${ }^{18}$

The ultrasound characteristics selected in this study were based on criteria that have been used by other researchers to describe more organised effusions. ${ }^{15-17}$ We refined previous variables seeking a more quantitative and objective measures of assessment, though this did not improve the utility of ultrasound to predict outcome. Despite efforts to standardise these criteria there remains a large subjective element in their description, as reflected by the variability in the kappa statistic. Jaffe et al ${ }^{15}$ reported better overall interobserver agreement in describing ultrasound pleural fluid by two radiologists (kappa 0.71) compared with our data (kappa $0.22-0.72$ ), although they employed a simplified grading system within a smaller sample size. Further, in that study interobserver agreement was lower when the same radiologists reported a more comprehensive description of CT findings in empyema (kappa 0.52). 
Our study design had several limitations. Firstly, the radiologists were asked to describe the pleural effusion from still images captured by another sonographer. This approach denied the radiologist the dynamic changes associated with probe positioning and respiratory movement. The consultants may have focussed on different still images from the patient to formulate their descriptions. This study also did not include the duration of symptoms at the time of the ultrasound and ultrasound changes may vary throughout the disease course.

Secondly, using retrospective data, the indications for reintervention were not standardised but interpreted from the clinical record. Most cases were associated with a persistent fever or a clinically significant air leak, these features could represent more complicated disease such as necrotising pneumonia and bronchopleural fistulae rather than a failure of drain efficacy. In these cases, no diagnosis of necrotising pneumonia was recorded, though in the absence of confirmatory CT imaging this is often a clinical diagnosis. Further research should consider this interaction as a potential confounding factor.

\section{Conclusion :}

In a large cohort of children managed with chest drainage and fibrinolytic therapy for paediatric empyema, we report hyperechoic fluid as a being associated with treatment failure. There was no association between pleural thickening, the number of septations, the size of the largest locule, and the maximal depth of an effusion or the interventional radiologist's estimated risk of reintervention. Even with objective criteria for assessment of pleural fluid, ultrasound imaging findings cannot predict the risk of treatment failure.

References

1. Bowen S-JM, Thomson AH. British Thoracic Society Paediatric Pneumonia Audit: a review of 3 years of data. Thorax. 2013;68(7):682-683.

2. Dorman RM, Vali K, Rothstein DH. Trends in treatment of infectious parapneumonic effusions in US children's hospitals, 2004-2014. Journal of pediatric surgery. 2016;51(6):885-890.

3. Segerer FJ, Seeger K, Maier A, et al. Therapy of 645 children with parapneumonic effusion and empyemaA German nationwide surveillance study. Pediatric pulmonology. 2017;52(4):540-547.

4. Balfour-Lynn IM, Abrahamson E, Cohen G, et al. BTS guidelines for the management of pleural infection in children. Thorax.2005;60(suppl 1):i1-i21.

5. Cobanoglu U, Sayir F, Bilici S, Melek M. Comparison of the methods of fibrinolysis by tube thoracostomy and thoracoscopic decortication in children with stage II and III empyema: a prospective randomized study.Pediatric Reports. 2011;3(4).

6. Marhuenda C, Barceló C, Fuentes I, et al. Urokinase versus VATS for treatment of empyema: a randomized multicenter clinical trial.Pediatrics. 2014;134(5):e1301-e1307.

7. Peter SDS, Tsao K, Harrison C, et al. Thoracoscopic decortication vs tube thoracostomy with fibrinolysis for empyema in children: a prospective, randomized trial. Journal of pediatric surgery.2009;44(1):106-111.

8. Sonnappa S, Cohen G, Owens CM, et al. Comparison of urokinase and video-assisted thoracoscopic surgery for treatment of childhood empyema.American journal of respiratory and critical care medicine.2006;174(2):221-227.

9. Pacilli M, Nataraja RM. Management of paediatric empyema by video-assisted thoracoscopic surgery (VATS) versus chest drain with fibrinolysis: systematic review and meta-analysis. Paediatric respiratory reviews. 2019;30:42-48.

10. Cohen E, Weinstein M, Fisman DN. Cost-effectiveness of competing strategies for the treatment of pediatric empyema. Pediatrics.2008;121(5):e1250-e1257.

11. Kelly MM, Coller RJ, Kohler JE, et al. Trends in hospital treatment of empyema in children in the United States. The Journal of Pediatrics. 2018;202:245-251. e241. 
12. Heuvelings CC, Bélard S, Familusi MA, Spijker R, Grobusch MP, Zar HJ. Chest ultrasound for the diagnosis of paediatric pulmonary diseases: a systematic review and meta-analysis of diagnostic test accuracy.British medical bulletin. 2019.

13. Claes A-S, Clapuyt P, Menten R, Michoux N, Dumitriu D. Performance of chest ultrasound in pediatric pneumonia. European journal of radiology. 2017;88:82-87.

14. Calder A, Owens CM. Imaging of parapneumonic pleural effusions and empyema in children. Pediatric radiology. 2009;39(6):527-537.

15. Jaffe A, Calder AD, Owens CM, Stanojevic S, Sonnappa S. Role of routine computed tomography in paediatric pleural empyema. Thorax. 2008;63(10):897-902.

16. McHugh ML. Interrater reliability: the kappa statistic.Biochemia medica. 2012;22(3):276-282.

17. Huang H-C, Chang H-Y, Chen C-W, Lee C-H, Hsiue T-R. Predicting factors for outcome of tube thoracostomy in complicated parapneumonic effusion or empyema. Chest. 1999;115(3):751-756.

18. Kalfa N, Allal H, Lopez M, et al. Thoracoscopy in pediatric pleural empyema: a prospective study of prognostic factors. Journal of pediatric surgery. 2006;41(10):1732-1737.

\section{Hosted file}

Table 1 PPulm.docx available at https://authorea.com/users/443322/articles/543342-paediatricempyema-are-ultrasound-characteristics-at-the-time-of-intervention-predictive-oftreatment-failure

\section{Hosted file}

Table 2 PPulm.docx available at https://authorea.com/users/443322/articles/543342-paediatricempyema-are-ultrasound-characteristics-at-the-time-of-intervention-predictive-oftreatment-failure

\section{Hosted file}

Table 3 PPulm.docx available at https://authorea.com/users/443322/articles/543342-paediatricempyema-are-ultrasound-characteristics-at-the-time-of-intervention-predictive-oftreatment-failure

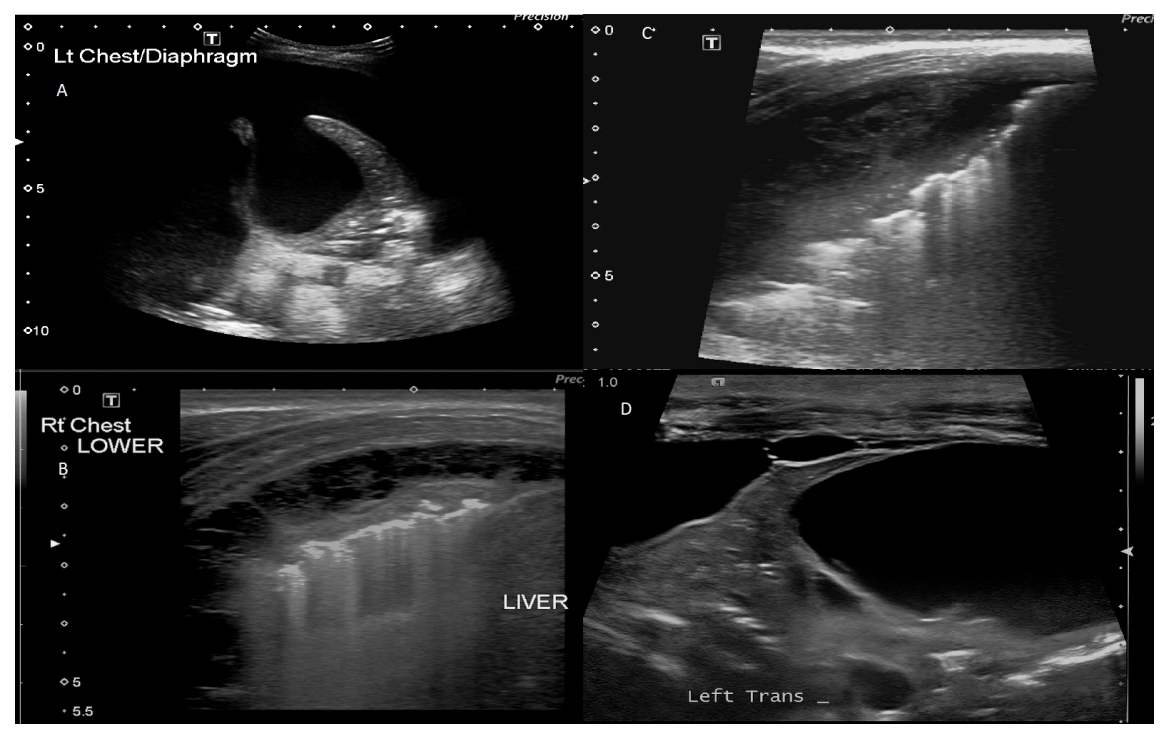

